\title{
Numerical Calculations for Nonlinear Characteristics of Chaotic Systems
}

\author{
Ma Shangang, Jin Fubao, Liang Bin, Tang Yan \\ School of Water Resources and Electric Power, Qinghai University, Xining, China \\ Email address: \\ msgqhu@139.com (Ma Shangang),jinfubao@163.com (Jin Fubao), 187101902@qq.com (Liang Bin), ty980231@163.com (Tang Yan)

\section{To cite this article:} \\ Ma Shangang, Jin Fubao, Liang Bin, Tang Yan. Numerical Calculations for Nonlinear Characteristics of Chaotic Systems. Journal of \\ Electrical and Electronic Engineering. Vol. 3, No. 5, 2015, pp. 150-155. doi: 10.11648/j.jeee.20150305.19
}

\begin{abstract}
This paper describes the relationship between Lyapunov index and the chaos of the dynamics system. Mainly describe the Lorenz system and Rossler system, and calculated the Lyapunov index of these two systems with nonlinear dynamics method. Analyses the iterative chaotic characteristic of Logistic system. And then calculate the Lyapunov index with the discrete system Lyapunov index computing method, and then calculated the correlation dimension.
\end{abstract}

Keywords: Lorenz System, Rossler System, Logistic System, Correlation Dimensions, Lyapunov Index

\section{混沌系统的非线性特征量的数值计算}

马山刚, 金福宝, 梁斌, 唐岩

水利电力学院, 青海大学, 西宁, 中国

\section{邮箱}

msgqhu@139.com(马山刚)，jinfubao@163.com(金福宝)，187101902@qq.com(梁斌)，ty980231@163.com(唐岩)

摘要: 本文简述了Lyapunov指数与系统的动力学混沌之间的关系。简要介绍了Lorenz系统与Rossler系统, 并用非线性 动力学的方法计算了其Lyapunov指数。分析了Logistic迭代映射的混沌特性, 用离散系统的Lyapunov指数计算法计算 其Lyapunov指数, 并计算了其关联维。

关键词: Lorenz系统, Rossler系统, Logistic系统, 关联维, Lyapunov指数

\section{1. 引言}

非线性科学是一门研究非线性现象共性的基础科学。 一般认为, 非线性科学的主体包括混沌（chaos）、分支 (bifurcation)、分形（fractal）、孤立子（soliton） 和复杂性（complexity） [1]。其中，混沌系统的基本特 点是运动对初值条件极为敏感, 两个靠近的初值所产生的 轨道随时间推移按指数方式分离 [2]。

\section{Lyapunov指数}

Lyapunov指数是衡量系统动力学特性的一个重要定 量指标 [3], 它表征了系统在相空间中相邻轨道间收敛或
发散的平均指数率。对于系统是否存在动力学混沌, 可以 从最大Lyapunov指数是否大于零非常直观的判断出来: 一 个正的Lyapunov指数, 意味着在系统相空间中, 无论初始 两条轨线的间距多么小, 其差别都会随着时间的演化而成 指数率的增加以致达到无法预测, 这就是混沌现象。

Lyapunov指数的和表征了椭球体积的增长率或减小 率, 对Hamilton系统, Lyapunov指数的和为零; 对耗散系 统, Lyapunov指数的和为负。如果耗散系统的吸引子是一 个不动点, 那么所有的Lyapunov指数通常是负的。如果是 一个简单的 $m$ 维流形 ( $m F 1$ 或 $m=2$ 分别为一个曲线或一个面)。 那么, 前 $m$ 个Lyapunov指数是零, 其余的Lyapunov指数为 负。不管系统是不是耗散的, 只要 $\lambda_{1}>0$ 就会出现混沌。 
微分动力系统Lyapunov指数的性质描述为, 对于一维 (单变量) 情形, 吸引子只可能是不动点（稳定定态）。 此时 $\lambda$ 是负的。对于二维情形, 吸引子或者是不动点或者 是极限环。对于不动点, 任意方向的 $\delta x_{i}$, 都要收缩, 故 这时两个Lyapunov指数都应该是负的。即对于不动点,

$\left(\lambda_{1}, \lambda_{2}\right)=(-,-)$ 。至于极限环, 如果取 $\delta x_{i}$ 始终 是垂直于环线的方向, 它一定要收缩, 此时 $\lambda<0$; 当取 $\delta x_{i}$ 沿轨道切线方向, 它既不增大也不缩小, 可以想象, 这时 $\lambda=0$ 。事实上, 所有不终止于定点而又有界的轨道（或吸 引子) 都至少有一个Lyapunov指数等于零, 它表示沿轨线 的切线方向既无扩展又无收缩的趋势。所以极限环的 Lyapunov指数是 $\left(\lambda_{1}, \lambda_{2}\right)=(0,-)$ 。

在三维情形下如表 1 所示 $[4]$ 。

\section{表1 三维 Lyapunov 指数与混沌吸引子的关系。}

\begin{tabular}{ll}
\hline$\lambda_{1}, \lambda_{2}, \lambda_{3}$ & 混沌吸引子 \\
\hline$(-,-,-)$ & 稳定不动点 \\
$(0,-,-)$ & 极限环; \\
$(0,0,-)$ & 二维环面 \\
$(+,+, 0)$ & 不稳极限环 \\
$(+, 0,0)$ & 不稳二维环面 \\
$(+, 0,-)$ & 奇怪吸引子 \\
\hline
\end{tabular}

李雅谱诺夫指数小于零, 则意味着相邻点最终要靠拢 合并成一点, 这对应于稳定的不动点和周期运动; 若指数 大于零, 则意味着相邻点最终要分离, 这对应于轨道的局 部不稳定, 如果轨道还有整体的稳定因素（如整体有界、 耗散、存在捕捉区域等），则在此作用下反复折叠并形成 混沌吸引子。指数越大, 说明混沌特性越明显, 混沌程度 越高。

\section{1. 连续系统Lyapunov指数谱的数值计算}

计算连续系统的Lyapunov指数谱即是求方程式 (1) [5]:

$$
\left\{\begin{array}{l}
\dot{x}=f(x) \\
\dot{\delta} \dot{x}_{1}=D f(x) \delta x_{1} \\
\vdots \\
\dot{\delta} \dot{x}_{m}=D f(x) \delta x_{m}
\end{array}\right.
$$

初值为 $\left(x(0)^{T}, \delta x_{1}(0)^{\mathrm{T}}, \cdots, \delta x_{\mathrm{m}}(0)^{\mathrm{T}}\right)$ 。若 $\delta x_{1}(0), \cdots, \delta x_{\mathrm{m}}(0)$ 线性无关, 则有式 (2)

$$
\frac{\left\|\delta x_{1}(t) \times \delta x_{2}(t) \times \cdots \times \delta x_{m}(t)\right\|}{\left\|\delta x_{1}(0) \times \delta x_{2}(0) \times \cdots \times \delta x_{m}(0)\right\|} \approx \exp \left[\left(\lambda_{1}+\lambda_{2}+\cdots+\lambda_{m}\right) t\right]
$$

理论上, 上述算法可以完成Lyapunov指数谱的计算, 但是实质上在数值计算过程中若 $\delta x_{j}(t) \rightarrow \infty$ 会产生数据
溢出, 若 $\delta x_{j}(t) \rightarrow 0$ 则会产生截断误差, 且 $\delta x_{j}(t)$ 之间夹 角会趋于 0 , 使得线性无关的条件得不到满足。因此, 采 用分段求解且正交化初值。

将时间 $t$ 分为 $k$ 段, 对于 $t \in[k T, k T+T]$, 将系统转化 为式 (3) 形式:

$$
\left\{\begin{array}{l}
\dot{x}=f(x) \\
\dot{u}_{1}^{(k)}=D f(x) u_{1}^{(k)} \\
\vdots \\
\dot{u}_{m}^{(k)}=D f(x) u_{m}^{(k)}
\end{array}\right.
$$

其中向量 $\left(u_{1}^{(k)}(k T), u_{2}^{(k)}(k T), \cdots, u_{m}^{(k)}(k T)\right)$ 是由 $\left(\delta x_{1}(k T), \delta x_{2}(k T), \cdots, \delta x_{\mathrm{m}}(k T)\right)$ 生成的一组单位正交向 量。

\section{2. 离散系统Lyapunov指数的数值计算}

对于离散系统（迭代系统）, 也可以类似地定义轨线 的 Lyapunov 指数。以一维迭代映射 $f: R \rightarrow R$ 为例, $x_{n+1}=f\left(x_{n}\right)$ 。

设研究的轨线是 $x=\left(x_{1}, x_{2}, \cdots, x_{n}, \cdots\right)$, 它的一条临 近轨线是 $y=\left(y_{1}, y_{2}, \cdots, y_{n}, \cdots\right)$ 。式中, $x_{n}, y_{n}$ 是第 $n$ 次 迭代值。第 $n$ 次迭代时两条轨线的距离为: $d_{n}=\left|y_{n}-x_{n}\right|$, 起始迭代时两条轨线的距离是: $d_{1}=\left|y_{1}-x_{1}\right|$ 。

定义轨线的Lyapunov指数为式 (4)

$$
\lambda=\lim _{n \rightarrow \infty} \frac{1}{n} \ln \frac{d_{n+1}}{d_{1}}
$$

其中 $\frac{d_{n+1}}{d_{1}}=\frac{d_{2}}{d_{1}} \frac{d_{3}}{d_{1}} \cdots \frac{d_{n+1}}{d_{n}}$

一维迭代映射简单, 为计算, 令 $y_{n}=x_{n}+\varepsilon$, 由于 $y$ 是 $x$ 的邻近曲线, $|\mathcal{E}|$ 远小于 1 , 由式 (4) 得

$$
d_{n}=\left|y_{n}-x_{n}\right|=|\varepsilon|
$$

$d_{n+1}=\left|y_{n+1}-x_{n+1}\right|$ 可由以下推导求出。

由迭代公式得式 (6)

$$
y_{n+1}=f\left(y_{n}\right)=f\left(x_{n}+\varepsilon\right)
$$

用泰勒级数展开, 并有 $|\varepsilon|<<1$, 则有式 (7)

$$
y_{n+1} \approx f\left(x_{n}\right)+\frac{d f\left(x_{n}\right)}{d x} \varepsilon=x_{n+1}+\frac{d f\left(x_{n}\right)}{d x} \varepsilon
$$

从而可得式(8) 


$$
d_{n+1}=\left|y_{n+1}-x_{n+1}\right|=\left|\frac{d f\left(x_{n}\right)}{d x}\right| \mathcal{E}|=| \frac{d f\left(x_{n}\right)}{d x} \mid d_{n}
$$

即式 (9)

$\frac{d_{n+1}}{d_{1}}=\frac{d_{2}}{d_{1}} \frac{d_{3}}{d_{1}} \cdots \frac{d_{n+1}}{d_{n}}=\left|\frac{d f\left(x_{1}\right)}{d x} \frac{d f\left(x_{2}\right)}{d x} \ldots \frac{d f\left(x_{n}\right)}{d x}\right|$

故式（10）

$$
\lambda=\frac{1}{n} \sum_{i=1}^{n} \ln \left|\frac{d f\left(x_{i}\right)}{d x}\right|
$$

计算时注意将暂态部分舍去再进行Lyapunov指数的 计算。

\section{3. 关联维}

现代分形维的最早一种形式由Hausdorff在1919年给 出。但是对于有复杂结构的混沌吸引子, 它的各部分的自 然测度分布很不均匀。Hausdoff维的定义中对各个划分单 元同等统计, 没有考虑自然测度的差别。为此人们对 Hausdorff维进行了推广, 其中就有关联维。关联维也称 相关维, 它表示系统在多维空间中的疏密程度。它最吸引 人的地方是它可以相对容易地从实验数据中估计出来。关 联维的表达式为式 (11) [6]:

$$
D_{2}=\lim _{\varepsilon \rightarrow 0}\left[\frac{\ln \sum_{i} \mu^{2}\left(C_{i}\right)}{\ln \mathcal{E}}\right]
$$

其中 $\varepsilon$ 为一正常数, $\mu$ 为自然测度, $C_{i}$ 为划分单元。

$$
C(2, r)=\lim _{n \rightarrow \infty} \frac{1}{n^{2}} \sum_{j=1}^{n} \sum_{i=1}^{n} U\left(r-\left\|x_{i}-x_{j}\right\|\right)
$$

其中

$$
U(x)=\left\{\begin{array}{l}
1, x>0 \\
0, x<0
\end{array}\right.
$$

当 $\varepsilon \rightarrow 0$ 时, 有 $\frac{\ln C(\varepsilon)}{\ln \varepsilon} \rightarrow D_{2}$, 因此可以通过绘出 $\ln C(\varepsilon)-\ln \varepsilon$ 关系曲线, $\varepsilon$ 较小时, 曲线斜率可以估计 $D_{2}$ 。

\section{4. 连续系统的Lyapunov指数}

\subsection{Lorenz系统}

大气动力系统是用非线性偏微分方程描述的无穷维 动力系统, 其中, 平流项是最主要的非线性项。直接研究 大气动力系统难度极大, 通常是采用截谱法将系统近似地 转化为某有限维正交函数空间的由非线性常微分方程描 述的动力系统, 通过定性地研究该非线性常微分系统在相 空间的轨迹特性来阐明大气系统的动力学行为与平流项
的非线性效应, 以获得与实际基本相符的近似结果。世界 著名的气象物理学家Lorenz1961年在研究热对流不稳定 时, 采用高截谱方法由Saltrman给出的描写对流的控制方 程得到了被命名为Lorenz系统的 3 维非线性常微分方程, 首创性地发现了气象系统存在奇特的 “蝴蝶效应” , 揭示 了任何具有非周期行为的物理系统均将存在不可预期的 现象 [7]。从此揭开了研究混沌的新纪元, 起到里程碑的 作用。它的重大科学意义和应用价值是众所周知的。

$$
\left\{\begin{array}{l}
\dot{x}=-\sigma x+\sigma y \\
\dot{y}=-x z+R x-y \\
\dot{z}=x y-b z
\end{array}\right.
$$

$x, y, z$ 分别表示与对流强弱, 对流引起的水平温 差和垂直方向温度有关的变量。 $\sigma 、 R 、 b$ 分别表示普兰 多数、瑞利数和容器大小有关的参数。

当 $\sigma=16, R=45.92, b=4$ 时, 绘出系统的典型吸 引子如图1所示。选取 $T=0.1 \mathrm{~s}$, 舍去前 500 个迭代点, 绘 制Lorenz系统的Lyapunov指数谱如图2所示。从图中的稳 态过程可以容易得到该参数下的Lorenz 系统的三个 Lyapunov 指数为 $\left(\lambda_{1}, \lambda_{2}, \lambda_{3}\right)=(2.1643,-0.0000,-32.3851)$ 。 可以判断该参数下的Lorenz系统吸引子为奇异吸引子。

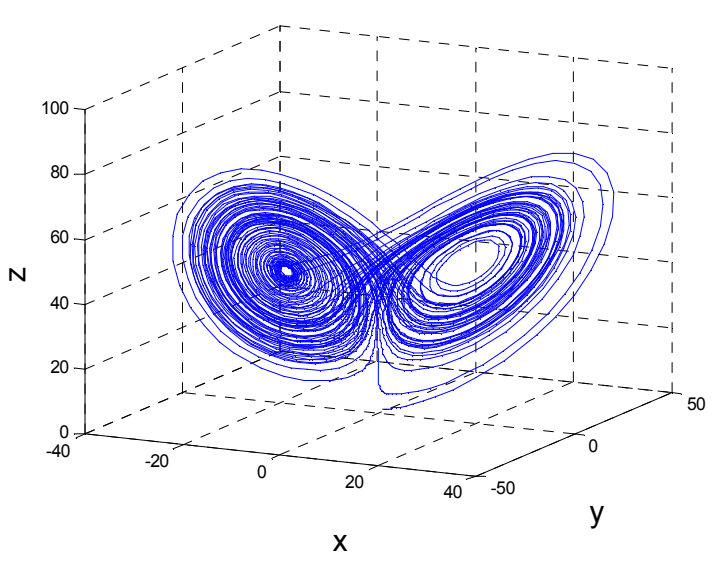

图1 Lorenz系统的典型吸引子。

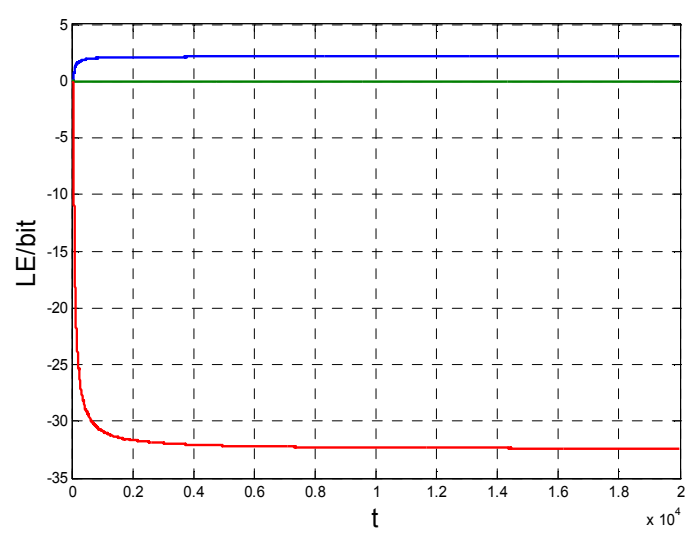

图2 Lorenz系统的Lyapunov指数谱。 


\subsection{Rossler系统}

自20世纪60年代以来, 在研究Lorenz系统的基础上, 许多研究者从数学, 物理等方面对混沌系统的特性进行了 深入的研究。其中德国物理化学家0. E. Rossler发现的 Rossler系统是由用两种不同的方法从Lorenz吸引子中抽 出的更简单、非堆成的吸引子结构, 只包含一个非线性项, 但其混沌动力学特性与Lorenz系统相似。

Rossler系统的动力学方程为式 (14) [8]:

$$
\left\{\begin{array}{l}
\dot{x}=-x-y \\
\dot{y}=x+a y \\
\dot{z}=b+x z-c z
\end{array}\right.
$$

取 $a=0.15, b=0.2, c=10$, 绘出系统的典型吸引 子如图3所示。选取 $T=0.1 s$, 舍去前 500 个迭代点, 绘制 Rossler系统的Lyapunov指数谱如图4所示。从图中的稳态 过程可以容易得到该参数下的 Rossler 系统的三个 Lyapunov指数为 $\left(\lambda_{1}, \lambda_{2}, \lambda_{3}\right)=(0.1278,0.0002,-14.1036)$ 。可 以判断该参数下的Rossler系统吸引子为奇异吸引子。

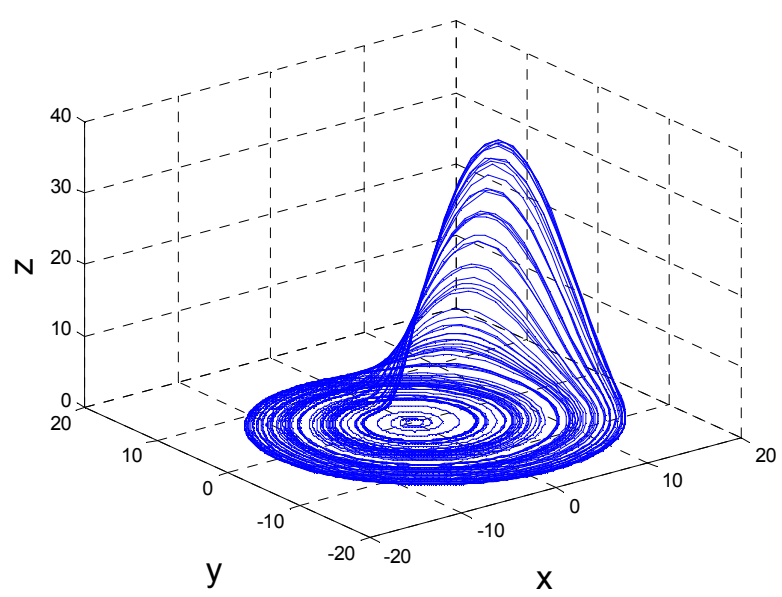

图3 Rossler系统的典型吸引子。

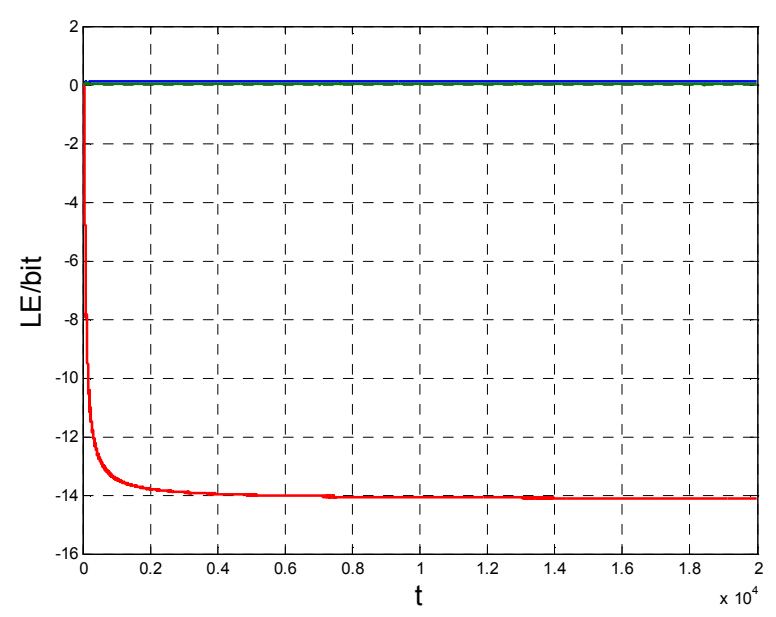

图4 Rossler系统的Lyapunov指数谱。

\section{5. 离散系统（Logistic系统）的非线性特征量}

\section{1. Lyapunov指数}

Logistic映射又称虫口模型, 是描述生物种群演化的 典型数学模型 [9] [10]。最初的Logistic 映射形式为: $x_{n+1}=x_{n}\left(a-b x_{n}\right)$ 。为了数学上处理方便, 取 $a=b=\mu$, 可得式(15)

$$
x_{n+1}=\mu x_{n}\left(1-x_{n}\right)
$$

混沌研究对非线性动力学的发展起着全局性和本质 性的影响, 非线性动力学的研究一开始就与混沌探索联系 在一起。Logistic映射是一维非线性映射,一维非线性映 射对研究耗散系统中的混沌具有普遍而重要的意义。在一 维非线性映射总得到的分岔序列与临界点附近的标度性 质对于高危的情况也普遍适用。数学上已经证明, 维耗散 系统的倍周期分岔所表现出来的标度性与一维非线性映 像是一样的原因可能在于, 在参数空间中某一点的邻域内 考察系统的变化时, 相空间的行为往往只在一维方向上发 生拓扑变化。因此, Logistic映射是非线性动力系统研究 中的基础映射之一。

图5给出了 $\mu$ 从2.5到4变化时, Logistic系统的分岔 图。从图中可以看出, 系统在 $\mu$ 为 3 时开始出现了分岔。 随着 $\mu$ 取值的变化, 系统逐渐进入混沌。
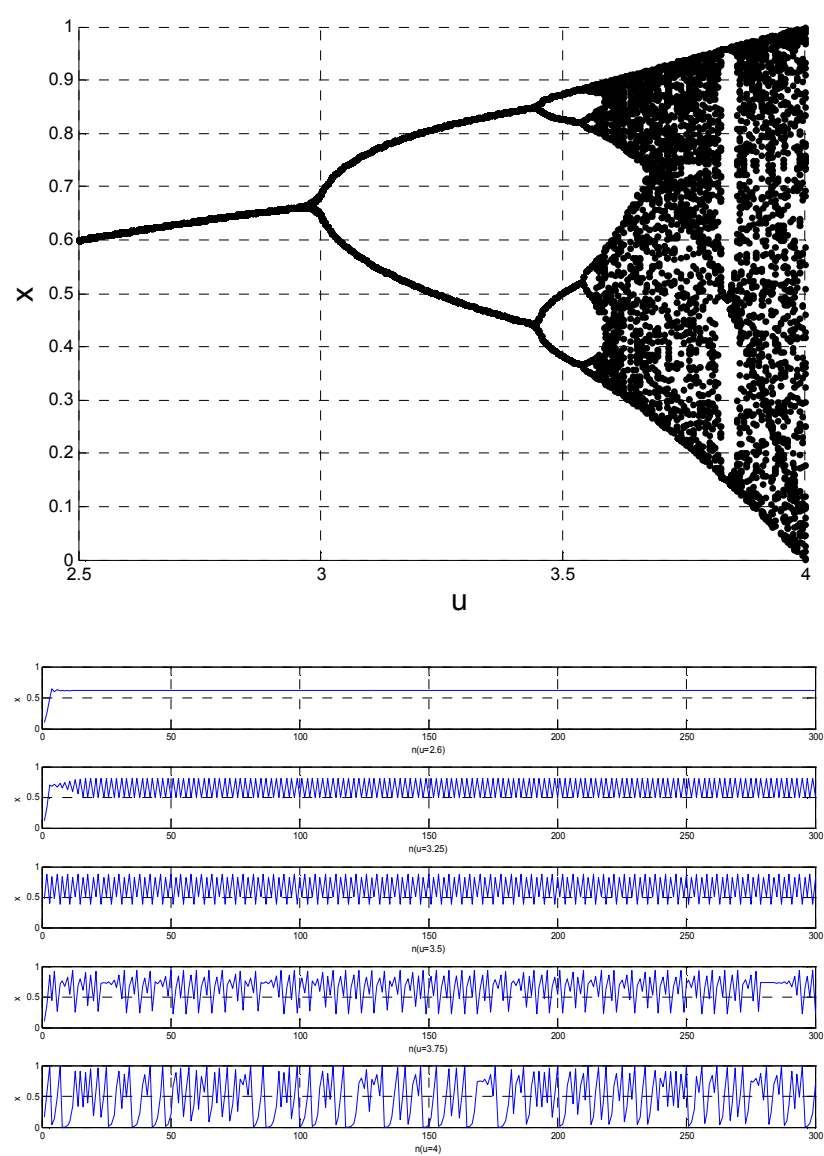

图5 Logistic系统的分岔图。 
本文对 $\mu$ 从 1 到 4 变化的过程中, 计算了系统的 Lyapunov指数, 绘于图6。从图中也可以看出, 在 $\boldsymbol{\mu}$ 为 3 时, 系统的Lyapunov指数第一次超过了 0 , 因此开始出现 混沌。将 $\mu$ 值较大的部分放大, 如图7所示, 我们可以看 到更细节的部分。

结合图5-图7, 可以得到以下结论:

1) $\mu \in(1,3]$ 时, $\lambda<0$, 系统收玫, 其中 $\mu$ 为 2 时, 存在一个超稳定点, 因此 $\lambda \rightarrow-\infty$ (图中无法画出, 故有 间断）;

2) $\mu \in(3,3.571448]$ 时, 系统处于倍周期分岔, $\lambda$ 处 于 $0 \rightarrow-\infty \rightarrow 0$ 的循环过程;

3) $\mu \in(3.571448,3.82842]$ 时, 系统为阵发混沌状态, $\lambda$ 时而大于 0 , 时而小于 0 ;

4) $\mu \in(3.9,4]$ 时, $\lambda>0$ (图中小于 0 是计算精度造成), 系统处于混沌状态。

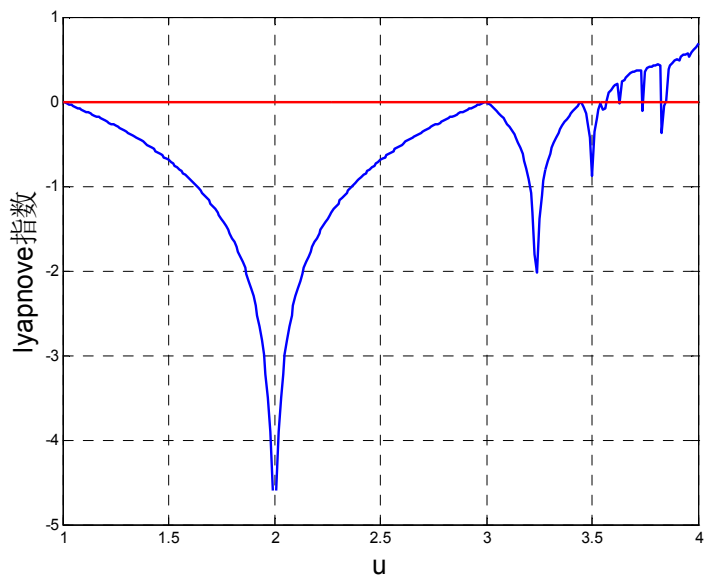

图6 Logistic系统的Lyapunov指数。

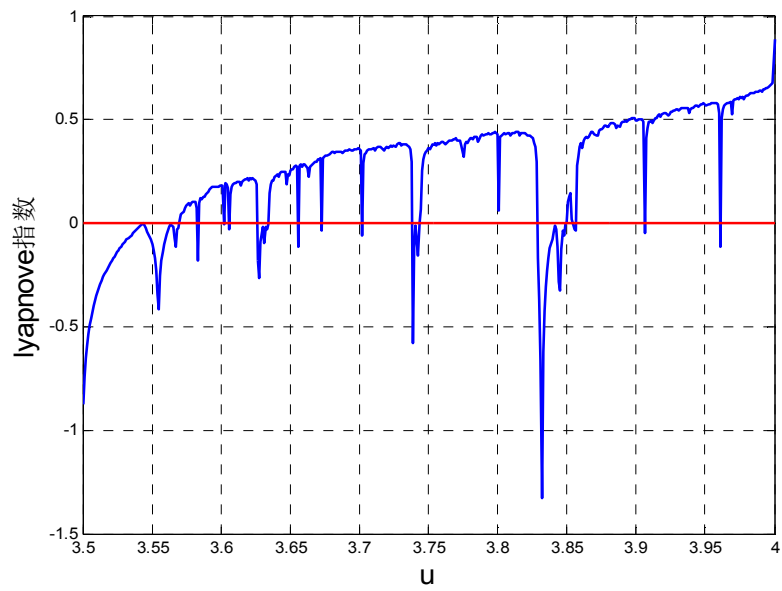

图7 图6的局部放大。

\section{2. Logistic系统的关联维}

绘出 $\ln C(\varepsilon)-\ln \varepsilon$ 关系曲线, 可以发现, 在 $\varepsilon$ 较小的 时候有噪声及饱和现象, 在 $\mathcal{E}$ 较大时亦有严重的饱和, 所
以欲求取 $D_{2}$, 仅需要对曲线的中间线性区进行最小二乘法 拟合。得一次项系数 $D_{2}$ 即为 0.7861 。

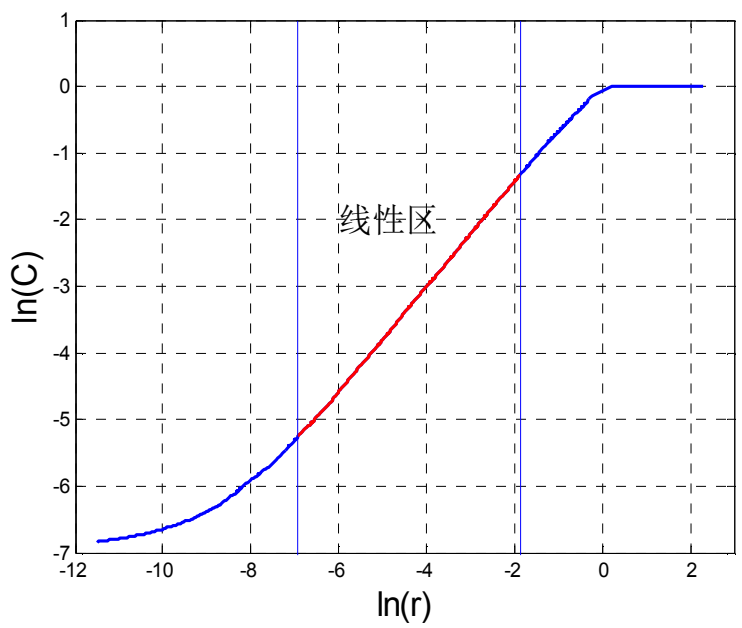

图5 Logistic系统 $\ln C(\varepsilon)-\ln \varepsilon$ 关系图。

\section{6. 结束语}

混沌系统的基本特点是运动对初值条件极为敏感，两 个靠近的初值所产生的轨道随时间推移按指数方式分离。 基于Lyapunov指数与系统的动力学混沌之间的密切关系, 本文采用非线性动力学的方法, 计算了Lorenz系统与 Rossler系统的Lyapunov指数; 采用离散系统的Lyapunov 指数计算法计算了Logistic系统的Lyapunov指数, 并计算 了其关联维。这对研究混沌系统具有重要意义。

\section{参考文献}

[1] 洪奕光. 非线性控制系统的若干分析和综合方法 [J]. 系统 科学与数学. $2012,12: 1497-1503$ 。

［2］伊利亚 - 普里戈金. 确定性的终结: 时间、混沌与新自然法 则 $[\mathrm{M}]$. 上海科技教育出版社. 2009。

[3] 高思莉, 肖青, 齐欣, 等. 逻辑斯蒂映像混沌区的几个非线性 特征量 $[J]$. 新乡师范高等专科学校学报, 2003, 02:10-14。

[4] 赫希. 微分方程、动力系统与混沌导论 [M]. 人民邮电出版 社, 2008。

[5] 李尧亭, 蔡诗东. 混沌和李雅谱诺夫特征指数 $[J]$. 物理, 1996, 05 (5):0-0。

[6] King C C. Fractal and chaotic dynamics in nervous systems $[J]$. Progress in Neurobiology, 1991, $36(4): 279-308$ 。

[7] 廖晓昕, 罗琦. Lorenz混沌系统Lyapunov稳定性简洁的代数 充要条件及其应用 $[\mathrm{J}]$. 中国科学：信息科 学, 2010, 08 (8) : 1086-1095。 
[8] 尤晓玲. Rossler 系统的动力学行为研究及混沌抑制 [J]. 工业仪表与自动化装置, 2013, 04:6-8。

[9] 王云雄, 翁贻方, 郑德玲. 混沌的复杂度研究方法和 Logistic 映射分析 $[\mathrm{J}]$. 北京工商大学学报: 自然科学 版, 2006, 2:38-41。
[10] 王立明.二维logistic映射的动力学行为和奇怪吸引子的 分形特征 [J]. 大学物理, 2010, 2:21-25。 